\title{
Nota técnica \\ MOVILIDAD DE METALES DEL SUELO AL PASTO EN LA REGIÓN NORTE DE COSTA RICA
}

\author{
Alfonso Salazar-Matarrita ${ }^{1 / *}$, Mario Cubero-Campos², Bárbara Durán-Jiménez ${ }^{3}$ \\ Palabras clave: Fluorescencia de rayos X; metales pesados; suelo; pasto. \\ Keywords: X-ray Fluorescence; heavy metals; soil; grass. \\ Recibido: $21 / 05 / 19$ \\ Aceptado: 13/08/19
}

RESUMEN

El estudio se basó en medir la concentración de metales en el suelo y en el pasto circundante de variedades utilizadas en la zona (Kikuyocloa clandestinum y Brachiara Brizantha) para determinar su movilidad, medido como porcentaje de transferencia del suelo al pasto. Se seleccionaron sitios de la región norte de Costa Rica, dividida en Región Chorotega y Región Huetar Norte. Se identificaron y compararon las concentraciones de potasio $(\mathrm{K})$, calcio $(\mathrm{Ca})$, manganeso $(\mathrm{Mn})$, hierro $(\mathrm{Fe})$, cobre $(\mathrm{Cu})$ y zinc (Zn). Se experimentó con la movilidad de estos elementos del suelo al follaje del pasto, por su importancia como nutrimentos en la fertilidad de los suelos y del follaje del pasto. Los elementos $\mathrm{Mn}, \mathrm{Fe}, \mathrm{Cu}$ y $\mathrm{Zn}$ se consideran metales pesados y en las zonas estudiadas no constituyen contaminantes. Se utilizó la técnica de fluorescencia de rayos $\mathrm{X}$ dispersiva en energía (FRXDE), para determinar la composición elemental en las matrices de suelo y del follaje del pasto, por ser

\footnotetext{
* Autor para correspondencia. Correo electrónico: alfonso.salazar@ucr.ac.cr

1 Universidad de Costa Rica, Centro de Investigaciones en Ciencias Atómicas, Nucleares y Moleculares (CICANUM), Costa Rica. (D) 0000-0002-6492-3029.
}

\begin{tabular}{|c|c|}
\hline \\
\hline \multicolumn{2}{|c|}{$\begin{array}{l}\text { Mobility of metals from the soil to the } \\
\text { grass in the northern region of Costa Rica. The } \\
\text { study was based on measuring the concentration } \\
\text { of metals in the soil and in the surrounding } \\
\text { grass varieties used in the area (Kikuyocloa } \\
\text { clandestinum and Brachiara Brizantha) to } \\
\text { determine their mobility, measured as a } \\
\text { percentage of soil transfer to the grass. Sites from } \\
\text { the northern region of Costa Rica were selected, } \\
\text { divided into Chorotega Region and Huetar Norte } \\
\text { Region. The concentrations of potassium (K), } \\
\text { calcium (Ca), manganese (Mn), iron (Fe), copper } \\
\text { (Cu) and zinc (Zn) were identified and compared. } \\
\text { It was experimented with the mobility of these } \\
\text { elements from the soil to the grass foliage, due to } \\
\text { its importance as nutrients in the fertility of soil } \\
\text { and grasses. Mn, Fe, Cu and Zn are considered } \\
\text { heavy metals and in the studied areas do not } \\
\text { constitute contaminants. The energy dispersive } \\
\text { X-ray fluorescence technique (FRXDE) was } \\
\text { used to determine the elementary composition }\end{array}$} \\
\hline & $\begin{array}{l}\text { Universidad de Costa Rica, Escuela de Física y } \\
\text { Centro de Investigaciones en Ciencias Atómicas, } \\
\text { Nucleares y Moleculares (CICANUM), Costa Rica. } \\
\text { (DD 0000-0002-5183-4668. }\end{array}$ \\
\hline & $\begin{array}{l}\text { Universidad de Costa Rica, Centro de Investigaciones } \\
\text { en Ciencias Atómicas, Nucleares y Moleculares } \\
\text { (CICANUM), Costa Rica. }\end{array}$ \\
\hline
\end{tabular}


una técnica no destructiva y de análisis elemental múltiple y simultáneo. Al comparar las concentraciones de $\mathrm{K}$ se encontraron valores superiores en el pasto que en el suelo, por lo que se descartó calcular la movilidad con la metodología definida en este trabajo. Para los restantes elementos se calculó la movilidad como el porcentaje de transferencia de los metales al pasar del suelo al pasto. Los resultados obtenidos muestran una transferencia promedio moderada para el Ca $(37,04 \%)$ y el $\mathrm{Zn}(24,75 \%)$, y una baja transferencia para el $\mathrm{Mn}(1,85 \%), \mathrm{Fe}(1,35 \%)$ y $\mathrm{Cu}(4,35 \%)$.

\section{INTRODUCCIÓN}

El pasto, considerado como varias especies de gramíneas, constituye una base de la alimentación del ganado vacuno, tanto en la producción de leche y sus derivados como en el engorde. Determinar la concentración de los elementos metálicos en el pasto, en especial los denominados metales pesados y el porcentaje de transferencia del suelo al pasto, constituye un factor relevante, para encontrar o descartar posible contaminación que afecte la alimentación y la salud animal y que adicionalmente incida en la reducción de la calidad de la alimentación humana en la leche de vaca, sus derivados y en la carne.

La calidad del suelo, donde se encuentra sembrado el pasto, es fundamental para determinar el efecto que tienen ciertos metales al movilizarse del suelo al pasto. Durante el tratamiento de los suelos, en su preparación para la siembra de pasto, se pueden introducir ciertos nutrimentos, que beneficien la calidad del producto sembrado, sin embargo, debe tomarse en cuenta la posible incorporación de metales pesados y sus efectos en la calidad del pasto. in the soil and grass foliage matrices, as it is a non-destructive technique and multiple and simultaneous elemental analysis. When comparing $\mathrm{K}$ concentrations, higher values were found in grass than in soil, so it was ruled out to calculate the mobility with the methodology defined in this work. For the remaining elements, mobility was calculated as the percentage of transfer of metals when moving from the ground to the grass. The results obtained showed a moderate average transfer for $\mathrm{Ca}(37.04 \%)$ and $\mathrm{Zn}(24.75 \%)$, and a low transfer for Mn (1.85\%), $\mathrm{Fe}(1.35 \%)$ and $\mathrm{Cu}(4.35 \%)$.

En el presente trabajo se procedió a determinar los elementos que se localizan en suelo y en pasto, mediante un sistema de análisis multielemental simultáneo y no destructivo, conocido como fluorescencia de rayos $\mathrm{X}$ dispersiva en energía (FRXDE), donde la muestra de interés se somete a una irradiación en un tubo de rayos X, con blanco de plata (Ag). Este método permitió identificar 11 elementos en el suelo y de esos, 7 elementos fueron reportados en las muestras de pasto, mientras que 4 estuvieron por debajo de la cantidad mínima detectable (Minimum Detectable Amount, MDA) por tanto no se detectaron en la muestra. Para el proceso de preparación e irradiación de ambas matrices, se siguieron los criterios del protocolo establecido para el sistema FRXDE (Salazar et al. 2004).

Es importante señalar que en los suelos agrícolas, por la acción antropogénica, la concentración de los metales pesados puede ser aumentada por aplicaciones químicas tales como fertilizantes, herbicidas, pesticidas o por la aplicación de abono animal y la presencia de aguas residuales (Iyaka et al. 2012, Montagne et al. 2007).

La fertilidad de los suelos en Costa Rica estudiada por Méndez y Bertsch (2012), 
determinaron 7 elementos esenciales y de valor nutricional; es así como citan al calcio, magnesio, potasio; conjuntamente con los metales pesados manganeso, hierro, cobre y zinc. En estudios de metales pesados en suelo, se han utilizado niveles de referencia no contaminantes para el cobre $\left(34 \mathrm{mg} . \mathrm{kg}^{-1}\right)$ y para el $\mathrm{Zn}$ (109 mg.kg-1) (Pérez et al. 2000 y Miranda et al. 2008).

En el presente trabajo se calculó la concentración en $\%$ o en mg. $\mathrm{kg}^{-1}$, de los elementos $\mathrm{K}, \mathrm{Ca}, \mathrm{Mn}, \mathrm{Fe}, \mathrm{Cu}$ y Zn, presentes en las matrices de suelo y pasto y luego se calculó la movilidad utilizando estos valores. Con el sistema de fluorescencia de rayos $\mathrm{X}$ instalado en el Centro de Investigaciones en Ciencias Atómicas, Moleculares y Nucleares (CICANUM) no se logra detectar la radiación proveniente del magnesio en razón de su bajo número atómico $(\mathrm{Z}=12)$ y la baja energía de sus rayos $\mathrm{X}(1,253 \mathrm{keV})$.

La movilidad se calculó solo para 5 elementos ( $\mathrm{Ca}, \mathrm{Mn}, \mathrm{Fe}, \mathrm{Cu}$ y $\mathrm{Zn}$ ) como la razón porcentual de las concentraciones (\% de transferencia) de los elementos entre la matriz del pasto respecto a la matriz del suelo. Esto corresponde al porcentaje de transferencia de metales entre el suelo y el pasto, sin considerar la contribución externa que pueda recibir la planta. En el caso del potasio (K) se descartó el cálculo de la movilidad con la metodología utilizada en el presente trabajo.

Los metales pesados se investigaron con respecto al peligro generado por los efectos de la contaminación. En este estudio no se encontraron valores a nivel tóxico y se incluyen, por lo tanto en las plantas, como en otros seres vivos; se consideran elementos esenciales al ser componentes estructurales o catalizadores de los procesos bioquímicos de los organismos (Méndez y Bertsch 2012).

Se seleccionaron, para muestreo de suelo y pasto, un total de 30 sitios de la parte norte del territorio nacional (Figura 1), dividida en 2 regiones geográficas e identificadas como Región Chorotega y Región Huetar Norte. En ambas regiones, se escogieron para muestreo los terrenos dedicados a la ganadería con áreas de pastoreo mayores a 100 metros cuadrados.

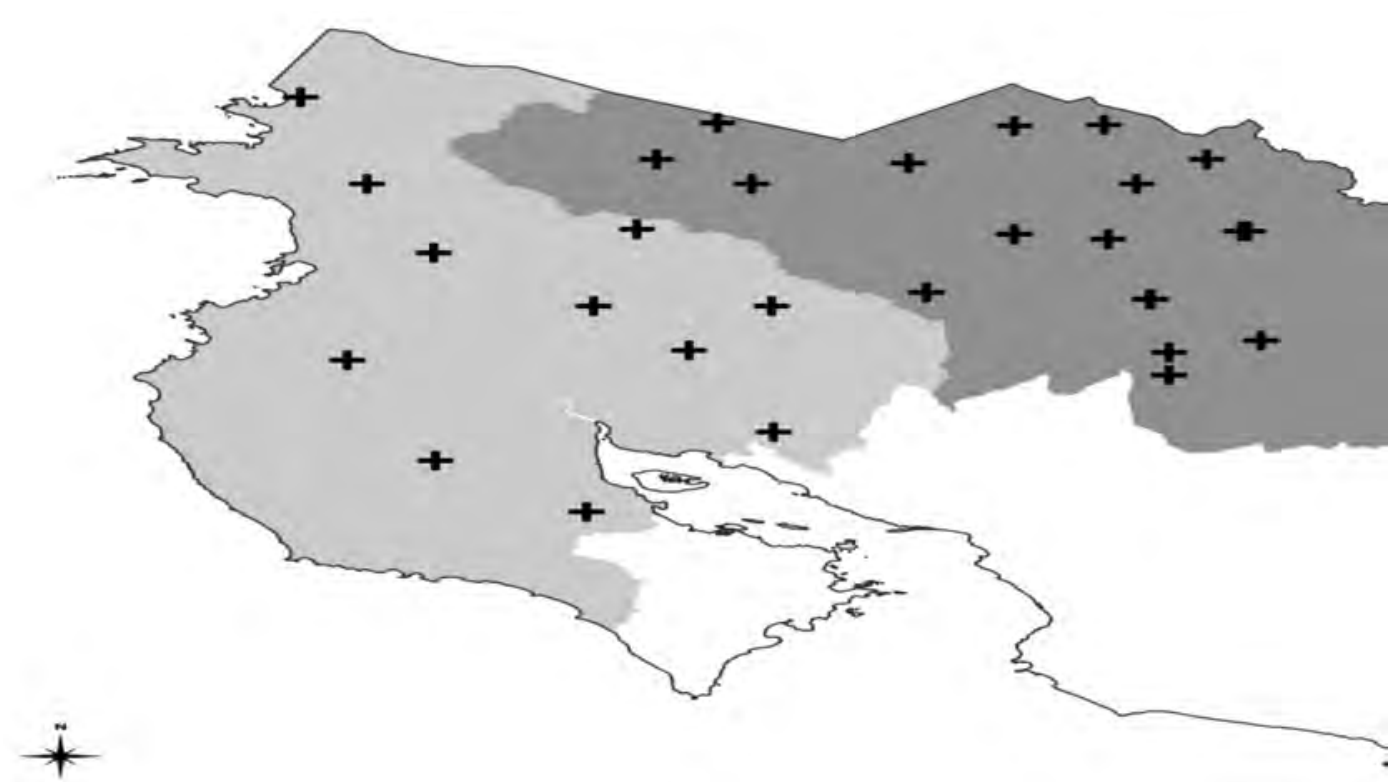

Figura 1 Distribución superficial de los puntos de muestreo en la Región Chorotega (gris claro) y la Región Huetar Norte (gris oscuro), que cubren cerca del $80 \%$ del área de la parte norte de Costa Rica. 
En la Región Chorotega se recolectaron muestras en potreros localizados en la provincia de Guanacaste, en los cantones de La Cruz, Liberia, Bagaces, Abangares, Tilarán, Carrillo, Nicoya y Nandayure y en la Región Huetar Norte se localizaron en la provincia de Alajuela y en los cantones de Upala, Los Chiles, Alajuela, Guatuso y San Carlos.

\section{MATERIALES Y MÉTODOS}

\section{Muestreo}

Se utilizaron bolsas de polietileno para obtener al menos $2 \mathrm{~kg}$ de muestra de suelo y de pasto. La toma de muestras se realizó en 30 sitios localizados en diferentes condiciones de suelo sembrados de pasto de la zona norte de Costa Rica. Cada posición es determinada mediante el uso de un sistema de posicionamiento global (Global Positioning System, GPS). La recolección del suelo se efectuó a una profundidad entre 10 y $20 \mathrm{~cm}$, luego de eliminar todo el material orgánico. De las muestras de pasto, se eliminaron las raíces y se conservó el follaje de la planta.

Los metales pesados se encuentran fuertemente ligados en los granos más finos del suelo. Su determinación se realizó en granos inferiores o iguales a $50 \mu \mathrm{m}$. A las muestras de suelo se les eliminó toda la materia orgánica. Se secaron al horno a una temperatura de $60^{\circ} \mathrm{C}$ durante $12 \mathrm{~h}$ para eliminar la humedad del material sin afectar la concentración de los metales (Salazar et al. 2004). En el caso de las de pasto, se secaron al horno a la misma temperatura pero por 24 horas.

Para el análisis con fluorescencia de rayos $\mathrm{X}$, se prepararon 3 pastillas por cada muestra de suelo y por cada muestra de pasto. Previamente, cada muestra se pulverizó en un mortero de ágata y se tamizó en cascada hasta conseguir un polvo fino con grano inferior o igual a $50 \mu \mathrm{m}$. Se depositó $1 \mathrm{~g}$ de polvo en un troquel de $13 \mathrm{~mm}$ y se comprimió en una prensa hidráulica a 6 toneladas hasta obtener la pastilla. En estas condiciones, la pastilla adquirió la condición de muestra gruesa, en la que el haz primario proveniente del tubo de rayos $\mathrm{X}$ se absorbe completamente en la pastilla. Para lograr la condición de muestra gruesa, se procedió a irradiar pastillas de distinto grosor al colocar un metal (i.e $\mathrm{Cu}$ ) detrás de la pastilla hasta lograr el grosor necesario para que desaparezca del espectro la radiación característica del metal utilizado. Para la muestra de suelo con una densidad de 1,9 g. $\mathrm{cm}^{3}$, se calculó un grosor mínimo de pastilla de $4 \mathrm{~mm} \mathrm{y,} \mathrm{para} \mathrm{el} \mathrm{pasto,}$ con una densidad aproximada a $0,94 \mathrm{~g} . \mathrm{cm}^{3}$, se calculó un grosor mínimo de $8 \mathrm{~mm}$.

Para calcular la concentración de los elementos de interés mediante la utilización del método BFP de parámetros fundamentales en fluorescencia de rayos X (Backscatter Fundamental Parameters, BFP) (Van Grieken et al. 2001), donde el 100\% de los rayos X característicos de cada elemento se obtuvo por la absorción del haz primario en el volumen de la muestra. Para disponer de una estadística básica, se calculó la concentración promedio de cada elemento a partir de la irradiación de las 3 pastillas preparadas por muestra.

\section{Sistema de irradiación, conteo y evaluación de la concentración}

El espectrómetro de rayos X (Figura 2) está conformado por un sistema de irradiación acoplado a un sistema de conteo de la intensidad de rayos $\mathrm{X}$, característicos de los elementos que componen la muestra de suelo o de pasto. La irradiación de los especímenes se realizó con un tubo de rayos X, AMPTEK Mini X, con blanco de plata $(E=22,1 \mathrm{keV})$, el cual se opera a $35 \mathrm{kV}$ y $15 \mu \mathrm{A}$, con un haz de rayos $\mathrm{X}$ que impactó la muestra en un ángulo de $67,5^{\circ}$ respecto a su superficie frontal. 




Figura 2 Sistema AMPTEK de tubo de rayos X con fuente de plata (Ag) y detector de silicio puro (Si) que se utiliza para la obtención del espectro de energía de la muestra de suelo o de pasto, preparada en pastilla.

Los rayos característicos, emitidos desde la muestra, se reciben en un detector de silicio puro, AMPTEK XR-100, localizado a un ángulo de $45^{\circ}$ respecto al haz de rayos $\mathrm{X}$ primario. El detector se une a un procesador de pulsos digital, AMPTEK PX4, que incorpora el analizador multicanal y la fuente de poder. Los espectros de rayos $\mathrm{X}$ se obtienen por medio del software AMPTEK XRF-FP, que visualiza los datos en computador $\mathrm{y}$, adicionalmente, realiza el cálculo de la concentración multielemental de la muestra. El análisis se basa en el método BFP, que permite obtener concentraciones simultáneas de los elementos de interés como referencia por medio de un material estandarizado. Para el caso del suelo, se utilizó el material estándar "Soil 7" (Cortes et al. 1990) suministrado por el Organismo Internacional de Energía Atómica (OIEA).

Para el caso del pasto, no se dispuso de un material de referencia directo, sin embargo, las concentraciones de los elementos se obtienen con el método BFP. La composición química del pasto presenta un número atómico promedio bajo, lo que aumenta la radiación Compton y modifica significativamente su relación con la radiación Rayleigh. Para establecer los ajustes pertinentes de la absorción de los rayos $\mathrm{X}$ primarios en la muestra de pasto, se midió en el espectro el aumento de la radiación Compton, producida por los elementos livianos, y se ajustó con el material de referencia de compuestos químicos conocidos y de número atómico promedio bajo, para proceder con el método BFP; de esta manera, se obtuvieron valores adecuados de la concentración de los elementos en el pasto.

\section{Movilidad y porcentaje de transferencia}

Para determinar la movilidad, se utilizó el porcentaje de transferencia de los elementos desde el suelo hacia el follaje del pasto, mediante la razón porcentual de la concentración (\% o $\mathrm{mg} \cdot \mathrm{kg}^{-1}$ ) de cada elemento en la muestra de suelo, sobre la concentración del mismo elemento en la muestra de pasto. Este porcentaje se expresa en la siguiente relación matemática: 
$\% \mathrm{TR}_{\mathrm{i}}=\mathrm{CP}_{\mathrm{i}} \times 100 / \mathrm{CS}_{\mathrm{i}}$

Donde:

$\% \mathrm{TRi}=$ porcentaje de transferencia.

$\mathrm{CP}=$ concentración en el pasto del elemento (i).

$\mathrm{CS}_{\mathrm{i}=}$ concentración del mismo elemento (i) en el suelo subyacente.

Esta metodología es aplicable cuando la concentración de los elementos en el pasto es inferior a la concentración en el suelo.

\section{RESULTADOS Y DISCUSIÓN}

La concentración de los 6 elementos de interés en el presente estudio ( $\mathrm{K}, \mathrm{Ca}, \mathrm{Mn}, \mathrm{Fe}, \mathrm{Cu}$, $\mathrm{Zn})$ se calculó para las muestras obtenidas de las Regiones Chorotega y Huetar Norte.

Los valores encontrados de las concentraciones de estos elementos, tanto en el suelo como en el pasto, no representan ningún peligro para la salud animal o humana. Actualmente, esta revisión debe enfocarse en señalar la posible contaminación por metales pesados en el sistema suelo-cosecha. (Rodríguez-Eugenio et al. 2018 y Prabhat et al. 2019).

Los resultados de las concentraciones de potasio (K), calculadas en las 30 muestras de pasto en ambas regiones, fueron superiores a las concentraciones calculadas en el suelo. En los ejemplares de pasto se obtuvieron concentraciones entre $0,95 \%$ y $7,5 \%$, y en las de suelo, del mismo sitio, las concentraciones oscilaron entre $0,07 \%$ y $0,9 \%$. Con estos resultados se descartó el cálculo de la movilidad del $\mathrm{K}$ utilizando la metodología establecida en el presente trabajo.

Las concentraciones de $\mathrm{Ca}$ en los sitios de Upala, San Carlos y Los Chiles de la Región Huetar Norte también tuvieron el mismo comportamiento y no se calculó el porcentaje de transferencia.

Estos resultados del potasio y el calcio, como nutrimentos esenciales en el crecimiento y fortalecimiento de las plantas, se podrían explicar como aplicaciones de agroquímicos al follaje para lograr una mejor fertilización foliar (Gutiérrez 2002 y Czarncki et al. 2015).

Para los restantes 5 elementos medidos, las concentraciones calculadas en el pasto no superaron las concentraciones encontradas en el suelo subyacente, lo cual permitió medir su movilidad al calcular el porcentaje de transferencia del suelo al pasto de cada elemento.

En la Tabla 1, se presentan los valores del promedio de las concentraciones en la Región Norte, para el que se consideran los datos obtenidos en la Región Chorotega y la Región Huetar Norte; estos se expresan en porcentaje (\%) para elementos mayores o menores ( $\mathrm{Ca}, \mathrm{Mn}$ y $\mathrm{Fe}$ ) y en mg.kg-1 para el caso de los elementos traza (Cu y $\mathrm{Zn})$. De acuerdo con los resultados obtenidos, la concentración de todos los elementos no presenta niveles de toxicidad.

Tabla 1. Promedio de las concentraciones $\left(\%\right.$ o mg. $\left.\mathrm{kg}^{-1}\right)$ en suelo y en pasto de los elementos estudiados, medido para la totalidad de las muestras de la región norte de Costa Rica y el porcentaje (\%) de transferencia entre el suelo y el pasto.

\begin{tabular}{lccccc}
\hline & $\mathrm{Ca}$ & $\mathrm{Mn}$ & $\mathrm{Fe}$ & $\mathrm{Cu}$ & $\mathrm{Zn}$ \\
\hline Suelo & $0,81 \%$ & $1,08 \%$ & $5,92 \%$ & $138 \mathrm{mg} \cdot \mathrm{kg}^{-1}$ & $101 \mathrm{mg} \cdot \mathrm{kg}^{-1}$ \\
Pasto & $0,30 \%$ & $0,02 \%$ & $0,08 \%$ & $6 \mathrm{mg} \cdot \mathrm{kg}^{-1}$ & $25 \mathrm{mg} \cdot \mathrm{kg}^{-1}$ \\
\%Transferencia & 37,04 & 1,85 & 1,35 & 4,35 & 24,75 \\
\hline
\end{tabular}

Para ambas regiones, se calculó el porcentaje de transferencia con respecto a cada uno de los elementos, así como su desviación estándar, que se incluyó para observar el grado de variabilidad en la región seleccionada. También se señalan los sitios donde se presentó la mayor y la menor movilidad, con el fin de visualizar el rango de los valores calculados, Tablas 2 y 3 . 
Tabla 2. Valores promedio y la desviación estándar del porcentaje de transferencia del suelo al pasto para cada uno de los elementos en la Región Chorotega.

Se indican los valores máximos y mínimos de la transferencia y los sitios donde se midieron.

\begin{tabular}{lccc}
\hline Elemento & $\begin{array}{c}\text { Transferencia promedio y } \\
\text { desviación estándar }(\%)\end{array}$ & $\begin{array}{c}\text { Máxima transferencia (\%) } \\
\text { y localización }\end{array}$ & $\begin{array}{c}\text { Mínima transferencia (\%) } \\
\text { y localización }\end{array}$ \\
\hline Calcio & $29,2 \pm 14,6$ & 49,7 (Liberia, CH03) & 4,6 (Tilarán, CH08) \\
Manganeso & $8,7 \pm 6,0$ & 24,4 (Liberia, CH04) & 2,2 (Abangares, CH07) \\
Hierro & $1,3 \pm 1,2$ & 3,5 (Nandayure, CH11) & 0,2 (Abangares, CH07) \\
Cobre & $4,5 \pm 0,5$ & 5,5 (Cañas, CH01) & 3,9 (Bagaces, CH05) \\
Zinc & $33,9 \pm 14,1$ & 67,0 (Tilarán, CH08) & 19,8 (Bagaces. CH06) \\
\hline
\end{tabular}

Tabla 3. Valores promedio y la desviación estándar del porcentaje de transferencia del suelo al pasto para cada uno de los elementos en la Región Huetar Norte.

Se indican los valores máximos y mínimos de la transferencia y los sitios donde se midieron.

\begin{tabular}{lccc}
\hline Elementos & $\begin{array}{c}\text { Transferencia promedio y } \\
\text { desviación estándar }(\%)\end{array}$ & $\begin{array}{c}\text { Máxima transferencia (\%) } \\
\text { y localización }\end{array}$ & $\begin{array}{c}\text { Mínima transferencia (\%) } \\
\text { y localización }\end{array}$ \\
\hline Calcio & $39,8 \pm 29,2$ & 90,1 (Upala, HN02) & 15,2 (Guatuso, HN12) \\
Manganeso & $27,2 \pm 23,6$ & 77,2 (Los Chiles, HN10) & 3,5 (Upala, HN01) \\
Hierro & $1,2 \pm 1,2$ & 4,3 (San Carlos, HN07) & 0,1 (Upala, HN03) \\
Cobre & $4,7 \pm 0,9$ & 6,8 (Upala, HN02) & 3,0 (Alajuela, HN13) \\
Zinc & $29,6 \pm 13,0$ & 68,6 (Los Chiles, HN05) & 11,8 (Upala, HN01) \\
\hline
\end{tabular}

El porcentaje de transferencia (\%) de cada elemento en función del sitio de recolección de la muestra se presenta para la Región Chorotega (Figura 3) y para la Región Huetar
Norte (Figura 4). En estas figuras, se escogió unir linealmente los puntos de los sitios, solo para visualizar el elemento al pasar de un sitio a otro. 


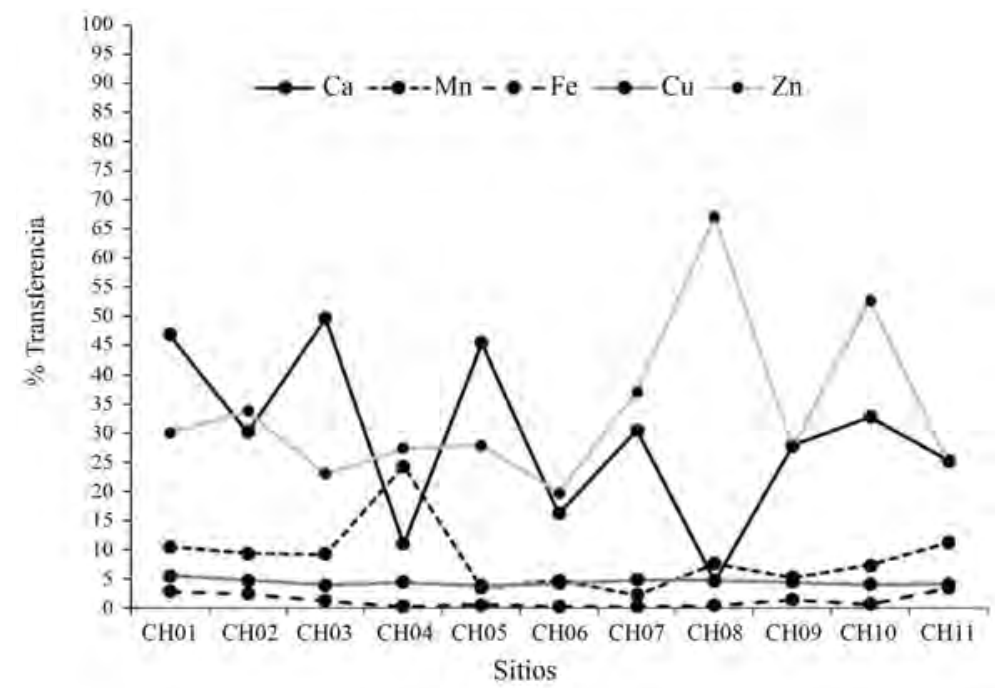

Figura 3 Porcentaje de transferencia (\%) de suelo a pasto para muestras de la Región Chorotega. Se incluyen los elementos $\mathrm{Ca}, \mathrm{Mn}, \mathrm{Fe}, \mathrm{Cu}$ y $\mathrm{Zn}$.

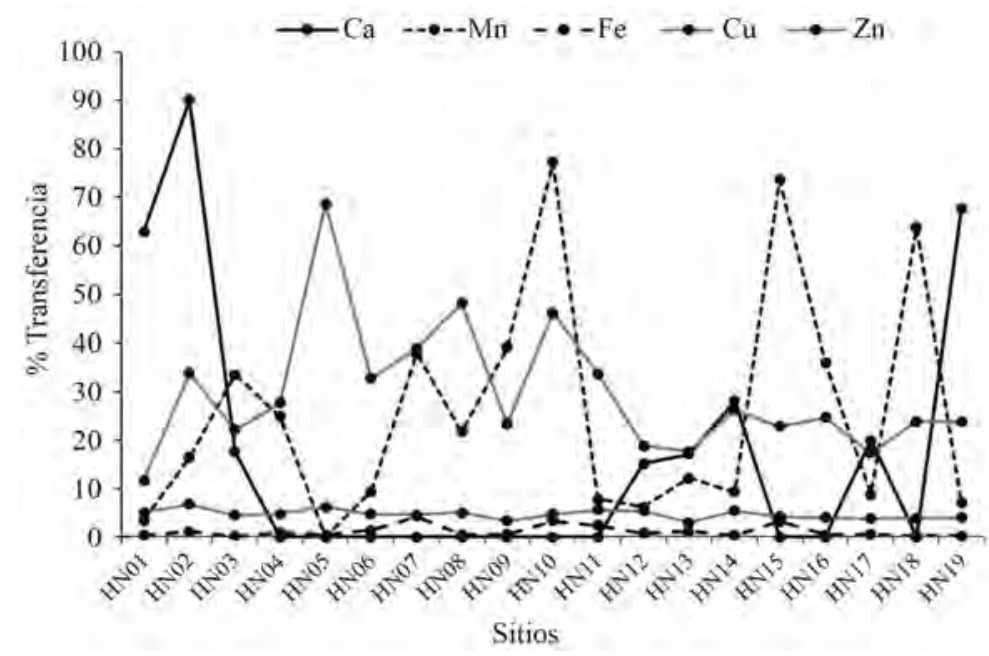

Figura 4 Porcentaje de transferencia de suelo a pasto para muestras de la Región Huetar Norte. Se incluyen los elementos Ca, $\mathrm{Mn}, \mathrm{Fe}, \mathrm{Cu}$ y $\mathrm{Zn}$. 
En la Región Chorotega se escogieron 11 sitios para la recolección de muestras en 9 cantones de la provincia de Guanacaste. Se escogieron 2 sitios en los cantones de Liberia y Bagaces para ampliar el área de cobertura del estudio. Los sitios se distribuyeron de la siguiente forma:

1. Cañas (sitio en Bebedero CH01).

2. La Cruz (sitio en Puerto Soley CH02).

3. Liberia (sitios $\mathrm{CH} 03, \mathrm{CH} 04$ ).

4. Bagaces (sitios en Bagaces $\mathrm{CH} 05$ y en Guayabo CH06).

5. Abangares (sitio en Las Juntas CH07).

6. Tilarán (sitio en Los Ángeles CH08).

7. Carrillo (sitio en Belén CH09).

8. Nicoya (sitio en Nicoya CH10).

9. Nandayure (sitio en Carmona CH11).

En la Región Huetar Norte se recolectaron muestras en 19 sitios de 5 cantones de la provincia de Alajuela. Se escogieron varios sitios por cantón en razón de las condiciones geográficas de la zona y se distribuyeron de la siguiente forma:

1. Upala (sitios en Canalete HN01; en Cartago Norte HN02; en San Ramón HN03, y en Nueva Esperanza HN04).

2. Los Chiles (sitios en El Jobo HN05; en Santa Fe HN06; en San Humberto HN10, y 2 en San Jorge HN11y HN19).

3. San Carlos (sitios en San Cristóbal HN07; en La Guaria HN08; en Aguas Zarcas HN14, y 2 sitios en Florencia HN15 y HN16).

4. Alajuela (2 sitios en Limoncito HN09 y HN18; un sitio en Esteritos HN13, y un sitio en Pocosol HN17).

5. Guatuso (sitio en Linda Vista de Venado HN12).

Es importante resaltar que en este tipo de suelos, que se emplean especialmente para la ganadería, se realizan aplicaciones de nutrimentos para el fortalecimiento de la calidad del pasto que se desarrolla y así beneficiar la alimentación de los animales por pastoreo, en especial, ganado de engorde, cría y producción de leche.

El flujo de nutrimentos, del suelo a la planta, es gobernado por una serie de procesos bioquímicos que se producen en su absorción que no fueron considerados en el presente trabajo (Antoniadis et al. 2015). Los resultados obtenidos mostraron que el calcio, el manganeso y el zinc varían significativamente de una región a otra, lo que determinó que el flujo es gobernado por situaciones diferentes, que dependen del tratamiento del suelo en cada región (Imunek et al. 2006). Los elementos $\mathrm{Fe}$ y $\mathrm{Cu}$ presentan una baja movilidad y semilar en ambas regiones, lo cual está asociado a la baja absorción de estos elementos en la planta (Salas 2002).

Se concluye que en las áreas estudiadas de la Región Norte, los porcentajes de transferencia promedio de metales constituyen un mecanismo general para evaluar su movilidad en sistema suelo-planta. Asimismo, la facilidad de su cálculo contribuye a guiar y valorar la necesidad de realizar una fertilización foliar de los cultivos de pasto para la alimentación animal, en especial, para los elementos estudiados.

\section{AGRADECIMIENTOS}

Se agradece el apoyo recibido por parte de la Vicerrectoría de Investigación de la Universidad de Costa Rica, del CICANUM-UCR y del trabajo técnico de los estudiantes universitarios Luis Fernando Umaña y Alonso Brenes.

\section{LITERATURA CITADA}

Antoniadis, V; Levizou, E; Ok, YS; Sebastian, A; Baum, Ch; Majeti, P; Wenzel, W; Rinklebe, J. 2017. Trace elements in the soil-plant interface: Phytoavailability, translocation, and phytoremediation. Earth-Science Reviews 171:621-645.

Cortes Toro, E; Parr, R; Clements, S. 1990. Biological and Environmental Reference Materials for Trace Elements, Nuclides and Organic Microcontaminants. Tec document, International Atomic Energy Agency. Viena, Austria, 114 p.

Czarncki, S; Düring, RA. 2015. Influence of long-term mineral fertilization on metal contents and 
properties of soil samples taken from different locations in Hesse, Germany. Soil Journal 1:23-33.

Gutiérrez, MV. 2002. Aspectos básicos de la nutrición mineral de las plantas, absorción foliar de sustancias útiles en la aplicación de agroquímicos al follaje. In Memoria, Seminario de Capacitación en Fertilización Foliar: Principios y Aplicaciones Meléndez, G; Molina, E (ed.). Laboratorio de Suelos y Foliares del Centro de Investigación Agronómica, Universidad de Costa Rica, San José, Costa Rica, p. 1-6.

Imunek, JJ; Sauvé, S; Adriano, D. 2006. Mechanisms and Pathways of Trace Element Mobility in Soils. Advances in Agronomy. Advances in Agronomy 91:111-178.

Iyaka, YA; Kakulu, SE. 2012. Heavy metal concentrations in top agricultural soils around ceramic and pharmaceutical industrial sites in Niger State, Nigeria. Research Journal of Environmental and Earth Sciences 4(2):171-176.

Méndez, JC; Bertsch, F. 2012. Guía para la interpretación de la fertilidad de los suelos de Costa Rica. Centro de Investigaciones Agronómicas, Universidad de Costa Rica. Asociación Costarricense de la Ciencia del Suelo. San José, Costa Rica. 106 p.

Miranda, D; Carranza, C; Rojas, C A; Martín, C; Fischer,G; Zurita, J. 2008 Acumulación de metales pesados en suelo y plantas de cuatro cultivos hortícolas, regados con agua del río Bogotá. Revista Colombiana de Ciencias Hortícolas 2:180-191.

Montagne, D; Cornu S; Bourennane, H; Baize, D; Ratié, C; King, D. 2007. Effect of Agricultural practices on trace-element distribution in soil. Commun. Soil Sci. Plant Anal. 38:473-491.

Prabhat, KR; Sang, SL; Ming, ZY; Fai, T; Ki-Hyun, K. 2019. Heavy metals in food crops: Health risks, fate, mechanisms, and management. Environment International 125:365-385.

Pérez, L; Moreno, AM; González, J. 2000. Valoración de la calidad de un suelo en función del contenido y disponibilidad de metales pesados. Edafología. U.C.M. 7-3:113-120.

Rodríguez-Eugenio, N; McLaughlin, M; Pennock, D. 2018. Soil Pollution: A hidden reality. Rome, FAO. 142 p.

Salas, RE. 2002. Herramientas de diagnóstico para definir recomendaciones de ferlización foliar. In Memoria, Seminario de Capacitación en Fertilización Foliar: Principios y Aplicaciones Meléndez, G; Molina, E (ed.). Laboratorio de Suelos y Foliares del Centro de Investigación Agronómica, Universidad de Costa Rica, San José, Costa Rica. p. 7-18.

Salazar, A; Lizano, O; Alfaro, E. 2004. Composición de sedimentos en las zonas costeras de Costa Rica utilizando Fluorescencia de Rayos-X (FRX). Revista Biología Tropical 52:61-75.

Van Grieken, N; Markowicz, A. 2001. Handbook of X-Ray Spectrometry. NY, USA, CRC PRES. p. 295-338. 